\title{
Characteristics of Prostate Cancers Missed by Biopsies: Evaluation of Cumulative Tumor Volume Missed According to Cancer True Prevalence*
}

\author{
Nicolas B. Delongchamps ${ }^{1 \#}$, Gustavo de la Roza ${ }^{2}$, Paul Perrin ${ }^{3}$, Michaël Peyromaure ${ }^{1}$, Gabriel P. Haas ${ }^{4}$ \\ ${ }^{1}$ Department of Urology, Cochin Hospital, Paris Descartes University, Paris, France \\ ${ }^{2}$ Department of Pathology, Upstate Medical University, Syracuse, USA \\ ${ }^{3}$ Department of Urology, Lyon South Hospital, Lyon, France \\ ${ }^{4}$ Astellas Pharma Global Development, Inc., Deerfield, USA \\ Email: "nicolasbdl@hotmail.com
}

Received May 14, 2013; revised June 16, 2013; accepted June 22, 2013

Copyright (C) 2013 Nicolas B. Delongchamps et al. This is an open access article distributed under the Creative Commons Attribution License, which permits unrestricted use, distribution, and reproduction in any medium, provided the original work is properly cited.

\begin{abstract}
Purpose: To characterize missed prostate tumors and their cumulative volume with various biopsy regimens to determine optimal biopsy schemes. Methods: We performed 6,12 and 18-core needle biopsies on 165 and 36-core biopsies on 47 autopsy prostates, respectively. The 6-core biopsy included 6 cores from the mid peripheral zone (MPZ), the 12-core biopsy included 6 cores from the MPZ and lateral PZ (LPZ), and the 18-core biopsies included 6 cores from the MPZ, LPZ and central zone (CZ). The 36-core biopsies included 12 cores in each of these 3 areas. We analyzed the sensitivity of biopsies at each site and evaluated the cumulative volume of cancers and tumor foci missed. Results: Whole-mount analysis identified 59 cancers, 110 tumor foci, and a total cumulative tumor volume of $43 \mathrm{~cm}^{3}$. The percentage of tumor foci and corresponding cumulative volume missed with $6,12,18$ and 36-core biopsies were of $79 \%$ and $58 \%, 64 \%$ and $48 \%, 57 \%$ and $26 \%$, and $42 \%$ and $17 \%$, respectively $(\mathrm{p}<0.05) .12$-core biopsies from the MPZ and LPZ performed best for clinically significant cancers detection. However, increasing the number of cores over the 6-core biopsy cutoff increased solely the detection of tumor foci $<0.5 \mathrm{~cm}^{3}$. Conclusion: Twelve biopsies from the MPZ and LPZ detected most of the clinically significant cancers while missing most of the tumor foci. These missed tumors represented only a small amount of the overall cancer volume.
\end{abstract}

Keywords: Prostate Cancer; Prevalence; Biopsy; Clinical Significance

\section{Introduction}

The trend among clinicians has been performed more prostate biopsies to detect more prostate cancers. Sextant biopsies have been all but abandoned, and most studies recommend extended biopsy protocols of 12 cores [1-3]. Although these efforts have undoubtedly led to increased detection of cancer, they have also led to the over diagnosis of small and well-differentiated tumors, designated as clinically insignificant $[4,5]$. Even undetected, they may not be of immediate threat, and should they be detected, not to be managed with radical and possibly morbid treatment. This issue recently came under increased

\footnotetext{
"Funding: National Institute on Aging (AG021389 to G.P.H.); National Cancer Institute (CA097751 to G.P.H.); National Institutes of Health (2U42RR006042-13). This study or its publication did not receive financial support from Astellas Pharma Global Development, Inc. ${ }^{\#}$ Corresponding author.
}

scrutiny as a consequence of the recent recommendations by the Task Force for Preventive Medicine [6]. Notwithstanding the evidence that PSA screening followed by early stage treatment may reduce mortality [7-9], they concluded that the use of PSA was harmful because it caused increased morbidity and over treatment of indolent tumors.

While recommendations from the Task Force for Preventive Medicine focused on the adverse effects of PSA screening, they did not address the role of prostate biopsy as a staging tool. Also, we have to be concerned not only with the ability of the biopsy to detect cancer, but also with the potential characteristics of cancers missed. We previously reported cancer detection rates obtained with different ex-vivo biopsy protocols performed on autopsied prostates [10]. Based on the cancer true prevalence, we found that 12 biopsies targeting the mid peripheral 
zone (MPZ) and lateral peripheral zone (LPZ) performed best for cancer detection. In the current study, we performed post hoc sub-analyses of our data to evaluate the performance of sextant, 12-core, 18-core and 36-core biopsies to detect cancer, with emphasis on the characterization of missed tumor foci and their cumulative volume.

\section{Method}

\subsection{Tissue Collection}

We prospectively collected 261 consecutive prostate glands from deceased men that were provided by the University Hospital, Syracuse, NY, the Onondaga County Medical Examiner, Syracuse, NY and by the National Disease Research Interchange, Philadelphia, PA. This study was approved by the Institutional Review Board, and the tissue suppliers obtained informed consent from the next of kin. The decedents had no known history of prostate cancer. At autopsy, the entire prostate gland, together with the seminal vesicles, were excised within 24 hours post-mortem by an experienced medical examiner or pathologist, en-block, and placed in $10 \%$ neutral buffered formalin. Prostatic tissue was not entirely removed in 49 (20\%) of the prostates autopsied; these subjects were excluded, leaving 212 prostate glands available for analysis.

\subsection{Prostate Biopsy}

All biopsies were performed in a manner that mimicked clinical biopsy with a standard $18 \mathrm{~F}$ spring-loaded biopsy gun. The needle was inserted through the posterior surface of the hand-held gland, and bilateral samples were taken from the apex, mid gland, and base. The first 165 autopsied glands were biopsied using an 18-core biopsy protocol (Figure 1). The first six cores, corresponding to the sextant biopsy protocol, were taken from the mid peripheral zone (MPZ). The next six cores were taken with the needle inserted into the central zone $(\mathrm{CZ})$. The last six cores were taken from the lateral peripheral zone (LPZ). The last 47 autopsied glands were biopsied using a saturation biopsy protocol of 36 cores: the first 18 cores were taken in a similar fashion and 6 additional cores were taken in each of the 3 locations (Figure 1).

\subsection{Whole Mount Prostate Processing and Histological Evaluation}

After the biopsies were taken, the glands were fixed in formalin for at least 72 hours. The glands were cut into 4-mm sections perpendicular to the posterior plane, labelled, embedded in paraffin, and further sectioned to produce $5-\mu \mathrm{m}$ whole-mount sections that were stained with hematoxylin and eosin. A single pathologist ana-

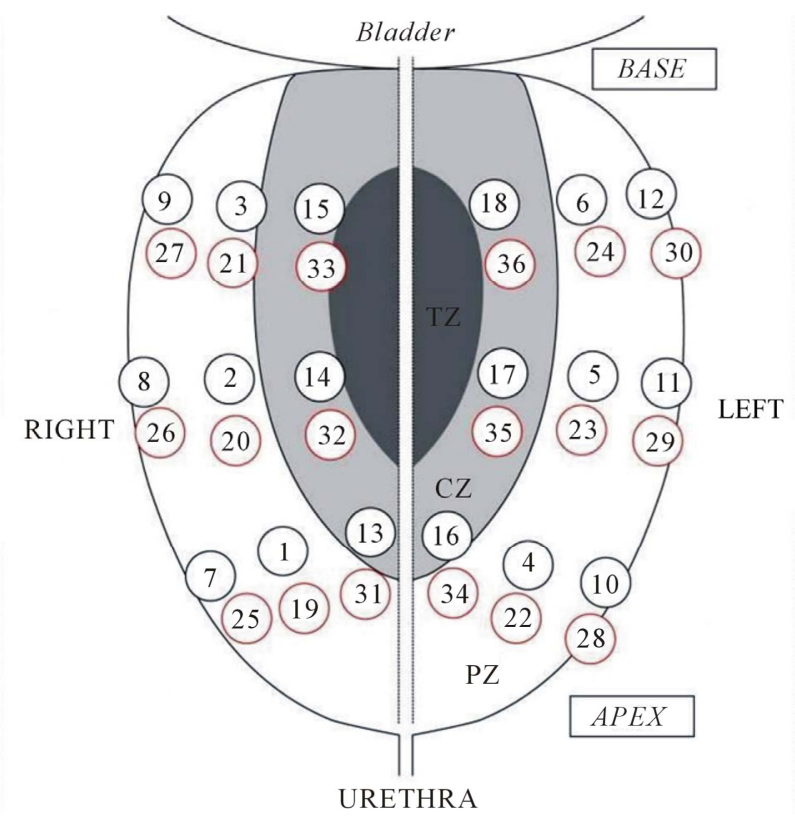

Figure 1. Schema of the anatomic sites of prostate biopsies taken from the 212 men postmortem. The first six biopsy cores (1-6) were taken from the mid peripheral zone (MPZ), the next six cores (7-12) from the central zone (CZ), and the last six cores (13-18) from the lateral peripheral zone (LPZ). In the last 47 glands, 6 additional biopsy cores were taken in each of these 3 mentioned areas.

lyzed the biopsies and whole-mount slides in a blinded fashion. The total number of tumor foci and their locations were recorded. An area of carcinoma was considered to be a separate focus if it was separated from the nearest adjacent focus by a low-power field diameter (4.5 $\mathrm{mm}$ ), as previously described [11]. Each tumor focus was graded according to the modified Gleason grading system [12].

\subsection{Digital Reconstruction and Measurement of Tumor Volume}

The surface of each tumor focus was determined by computerized planimetry, using an image analysis program $[10,13]$. Tumor volume was calculated by multiplying each tumor surface by the section thickness $(4 \mathrm{~mm})$ and by 1.5 to compensate for tissue shrinkage [14]. Tumors were considered clinically insignificant if they were organ-confined $(<\mathrm{pT} 3)$ with an index tumor volume of less than $0.5 \mathrm{~cm}^{3}$ and Gleason score 6 or less $[4,5]$. The cumulative tumor volume detected was calculated as the sum of the volumes of each individual tumor foci detected by biopsies. The cumulative cancer volume detected was calculated as the sum of the volumes of each cancer identified by biopsies.

\subsection{Statistical Methods}

Tumors missed by biopsies were compared with those 
detected using the student t-test and the $\chi^{2}$ test for quantitative and qualitative variables, respectively. McNemar and $\chi^{2}$ tests were used to compare the sensitivity of cancer detection, the number of missed foci and their cumulative volume between 6-, 12-, and 18- and 36-core biopsies. Sensitivity of biopsy results were calculated using as the standard either the presence of any prostate cancer or of clinically significant cancer on whole-mount analysis. All statistical tests were two-sided, and $\mathrm{P}$ values less than 0.05 were considered to be statistically significant. Statistical analyses were conducted using MedCalc ${ }^{\circledR}$.

\section{Results}

Pathologic evaluation of step sectioned prostates identified 59 prostates with cancer, and 110 tumor foci with a cumulative volume of $43 \mathrm{~cm}^{3}$. In the sub group of 47 autopsied glands biopsied with 36-core saturation biopsies, 12 cancers and 22 tumor foci were identified on step sectioned prostates, with a cumulative tumor volume of $4.7 \mathrm{~cm}^{3}$. Table 1 summarizes their characteristics and compares them between cancers detected and missed. Detection rates of significant and insignificant cancers were not statistically different between large $\left(>50 \mathrm{~cm}^{3}\right)$ and small $\left(\leq 50 \mathrm{~cm}^{3}\right)$ glands, whatever biopsy protocol tested $(\mathrm{p}>0.1)$.

Sextant prostate biopsies from the MPZ detected 18/59 $(30 \%)$ cancers and 23/110 (21\%) tumor foci. Of the 87 tumor foci missed, $7(8 \%)$ were $>0.5 \mathrm{~cm}^{3}, 19(22 \%)$ were $0.2-0.5 \mathrm{~cm}^{3}$ and $61(70 \%)$ were $<0.2 \mathrm{~cm}^{3}$. Thirteen $(15 \%)$ of the missed tumors were Gleason score $>6$. The cumulative tumor volume missed on sextant biopsy was of $25.1 \mathrm{~cm}^{3}(58 \%)$.

12-core biopsies from the MPZ and LPZ together detected $31 / 59(52 \%)$ cancers and 40/110 (36\%) tumor foci. Of the 70 tumor foci missed, $4(6 \%), 15(21 \%)$, and 51 $(73 \%)$ were $>0.5 \mathrm{~cm}^{3}, 0.2-0.5 \mathrm{~cm}^{3}$ and $<0.2 \mathrm{~cm}^{3}$, respectively. Nine $(13 \%)$ of the missed tumors were Gleason score $>6$. The cumulative tumor volume missed on 12 -core biopsies was of $20.6 \mathrm{~cm}^{3}(48 \%)$.

18-core biopsies from the MPZ, LPZ and CZ detected $32 / 59(54 \%)$ cancers and $47 / 110(43 \%)$ tumor foci. Of the 63 tumor foci missed, $3(5 \%), 13(21 \%)$ and $47(75 \%)$ were $>0.5 \mathrm{~cm}^{3}, 0.2-0.5 \mathrm{~cm}^{3}$ and $<0.2 \mathrm{~cm}^{3}$, respectively. Eight $(13 \%)$ of the missed tumors were Gleason score $>$ 6 . The cumulative tumor volume missed on 18-core biopsies was of $11.3 \mathrm{~cm}^{3}(26 \%)$ (Table 2).

36-core biopsies from the MPZ, LPZ and CZ detected $7 / 12(58 \%)$ cancers and 15/22 (68\%) tumor foci. Of the 7 tumor foci missed, $0,1(14 \%)$ and $6(86 \%)$ were $>0.5$ $\mathrm{cm}^{3}, 0.2-0.5 \mathrm{~cm}^{3}$ and $<0.2 \mathrm{~cm}^{3}$, respectively. One (14\%) of the missed tumors was Gleason score $>6$. The cumulative tumor volume missed on 36-core biopsies was of $0.8 \mathrm{~cm}^{3}(17 \%)$.

The sensitivity of 12-core biopsies for cancer detection was significantly higher than for sextant biopsies alone $(p<0.0009)$. The sensitivities of biopsies from the MPZ for clinically significant and insignificant cancer were $50 \%$ and $15 \%$, respectively, compared with $77 \%$ and $33 \%$ for those from the MPZ and LPZ combined ( $p=$ 0.02 and $p=0.04$, respectively). Increasing sampling over the 12-core cut-off decreased significantly the number of tumor foci missed as well as their cumulative volume, without increasing any further cancer detection rate (Figure 2). However, as shown in Figure 3, increased sampling over the 6-core cut-off decreased significantly the number of small tumor foci missed $\left(<0.5 \mathrm{~cm}^{3}\right)$ but not that of large $\left(>0.5 \mathrm{~cm}^{3}\right)$. The number of missed tumor foci with a Gleason score $>6$ decreased with additional biopsies, but the difference was not statistically significant $(p=0.2)$.

\section{Discussion}

In the large ongoing debate over prostate cancer screening, urologists mainly focus on cancers detected and their management. These cancers represent however only the tip of the iceberg. Estimation of tumors that are "missed" with particular biopsy regimens, the location of such tumors, and their histological characteristics and significance is therefore of critical importance.

Our analysis confirmed that performing more than 12 biopsies did not increase cancer detection any further. Although $64 \%$ of tumor foci and more than half (54\%) of the cancers present were missed by 12-core biopsies, they corresponded to a small amount of the overall cancer volume. The percentage of cumulative tumor volume missed with 12-core biopsies was high (48\%), but a significant number of the missed foci were located in glands diagnosed with cancer thanks to other contiguous or distant tumor foci detected by biopsies. As a result, only $14 \%$ of the overall cancer volume was truly missed with 12-core biopsies. Moreover, our results suggested that missed foci were significantly smaller than those detected and that they had a lower Gleason score. These findings are in accordance with earlier published studies suggesting that 12 cores directed to the peripheral zone of the prostate seem to have a high performance while minimizing the number of unnecessary cores [1-3]. Biopsies within the central zone have a low likelihood of detecting cancer in the absence of positive results from the peripheral zone of the gland $[1,15]$.

Once having recognized that 12 biopsies from the MPZ and LPZ performed best and detected most of the significant cancers, the next question would concern the need for additional biopsy sampling for cancer characterization. The potential benefit of additional biopsies would have to be balanced against the increased morbidity of the procedure. Biopsy-related morbidity is also a major issue highlighted by the Task Force for Preventive 
Table 1. Baseline characteristics of all patients and pathologic characteristics of prostate cancers.

\begin{tabular}{|c|c|}
\hline Characteristic & Median (IQR) or number (\%) \\
\hline \multicolumn{2}{|c|}{ All men $(\mathrm{N}=212)$} \\
\hline Age (years) & $64.5(54-73)$ \\
\hline Gland volume $\left(\mathrm{cm}^{3}\right)$ & $49(30-55)$ \\
\hline \multicolumn{2}{|l|}{ Race } \\
\hline White & $194(91 \%)$ \\
\hline Black & $9(4.5 \%)$ \\
\hline Hispanic & $1(0.5 \%)$ \\
\hline Unknown & $8(4 \%)$ \\
\hline \multicolumn{2}{|c|}{ Prostate cancers $(\mathrm{N}=59)$} \\
\hline \multicolumn{2}{|l|}{ Pathological stage } \\
\hline pT2a & $34(58 \%)$ \\
\hline pT2b & $3(5 \%)$ \\
\hline $\mathrm{pT} 2 \mathrm{c}$ & $14(24 \%)$ \\
\hline pT3a & $8(13 \%)$ \\
\hline \multicolumn{2}{|l|}{ Gleason score } \\
\hline$\leq 6$ & $41(69 \%)$ \\
\hline$\geq 7$ & $18(31 \%)$ \\
\hline \multicolumn{2}{|l|}{ Volume $\left(\mathrm{cm}^{3}\right)$} \\
\hline ITV & $0.222(0.074-0.530)$ \\
\hline TTV & $0.296(0.074-0.768)$ \\
\hline Tumor foci volume & $0.115(0.046-0.315)$ \\
\hline \multicolumn{2}{|l|}{ Clinical significance } \\
\hline Significant & $26(44 \%)$ \\
\hline Insignificant & $33(56 \%)$ \\
\hline \multicolumn{2}{|l|}{ Tumor foci location } \\
\hline MPZ only & $30(27 \%)$ \\
\hline LPZ only & $41(37 \%)$ \\
\hline MPZ and LPZ & $11(10 \%)$ \\
\hline $\mathrm{CZ}$ only & $24(22 \%)$ \\
\hline $\mathrm{MPZ}$ and $\mathrm{CZ}$ & $3(3 \%)$ \\
\hline $\mathrm{CZ}, \mathrm{MPZ}$ and LPZ & $1(1 \%)$ \\
\hline
\end{tabular}

Medicine [6] and has increased these past 10 years [16]. The rate of hospital admission within 30 days of having prostate biopsies was reported to be as high as $4 \%$, mainly for infection-related reasons [16]. In our analysis, 18 and 36-core biopsies allowed a significant increase in tumor foci detection and in their cumulative volume, improving therefore staging for each individual patient. However, this increased detection yielded only tumor foci $<0.5 \mathrm{~cm}^{3}$. Additionally, the number of missed poorly differentiated tumor foci (Gleason score $>6$ ) decreased with additional sampling, but the difference was not significant $(p=0.2)$. These findings support the concept that cancers are not missed by biopsies because of suboptimal technique, but rather because they are smaller and earlier in their development. In clinical practice, these cancers may be detected later with repeated biopsies [17]. Knowing the existence and characteristics of such secondary foci is of low value if a radical treatment is immediately intended. Conversely, to develop and increase the possibilities of active surveillance and focal therapies, performing an initial accurate cancer mapping of the prostate may be of crucial importance, especially when 12-core biopsies reveal only small amount of low grade cancer. This initial staging could then serve as a reference for potential re-biopsy strategies.

Our study has several limitations, the most important being the absence of pre-mortem PSA data available. PSA screening is indeed the first step before considering prostate biopsies. Another limitation is our "histological" definition of clinical significance, which did not take into consideration age, comorbidities, and other individual circumstances.

\section{Conclusion}

Twelve biopsies from the MPZ and LPZ detected most of 
Table 2. Pathological characteristics of cancers detected and missed with 18-core biopsies.

\begin{tabular}{|c|c|c|c|}
\hline & Prostate cancers & & \\
\hline & Detected $(\mathrm{n}=32)$ & Missed $(n=27)$ & $\mathrm{p}$ value \\
\hline Median (IQR) ITV $\left(\mathrm{cm}^{3}\right)$ & $0.485(0.265-0.845)$ & $0.081(0.047-0.215)$ & 0.02 \\
\hline Median (IQR) TTV $\left(\mathrm{cm}^{3}\right)$ & $0.638(0.333-1.057)$ & $0.087(0.051-0.215)$ & 0.005 \\
\hline \multicolumn{4}{|l|}{ Gleason score } \\
\hline$\leq 3+3$ & $19(59 \%)$ & $21(78 \%)$ & \multirow{2}{*}{0.2} \\
\hline$\geq 3+4$ & $13(41 \%)$ & $6(22 \%)$ & \\
\hline \multicolumn{4}{|l|}{ Number of foci } \\
\hline Unifocal & $9(28 \%)$ & $21(78 \%)$ & \multirow{2}{*}{0.0002} \\
\hline Multifocal & $23(72 \%)$ & $6(22 \%)$ & \\
\hline \multicolumn{4}{|l|}{ Significance } \\
\hline Significant & $21(66 \%)$ & $5(18 \%)$ & \multirow{2}{*}{0.0005} \\
\hline \multirow[t]{3}{*}{ Insignificant } & $11(34 \%)$ & $22(82 \%)$ & \\
\hline & Tumor foci & & \\
\hline & Detected $(n=47)$ & Missed $(n=63)$ & $\mathrm{p}$ value \\
\hline Median (IQR) tumor volume $\left(\mathrm{cm}^{3}\right)$ & $0.296(0.096-0.572)$ & $0.081(0.031-0.197)$ & 0.003 \\
\hline \multicolumn{4}{|l|}{ Number of tumor foci } \\
\hline Volume $\leq 0.2 \mathrm{~cm}^{3}$ & 20 & 47 & \\
\hline Volume $[0.2-0.5] \mathrm{cm}^{3}$ & 14 & 13 & \\
\hline Volume $\geq 0.5 \mathrm{~cm}^{3}$ & 13 & 3 & \\
\hline \multicolumn{4}{|l|}{ Gleason score } \\
\hline$\leq 3+3$ & 30 & 55 & \multirow{2}{*}{0.005} \\
\hline$\geq 3+4$ & 17 & 8 & \\
\hline \multicolumn{4}{|l|}{ Location } \\
\hline MPZ & $10(21 \%)$ & $20(32 \%)$ & 0.3 \\
\hline LPZ & $17(37 \%)$ & $24(38 \%)$ & 1 \\
\hline $\mathrm{CZ}$ & $7(15 \%)$ & $17(27 \%)$ & 0.2 \\
\hline $\mathrm{MPZ}+\mathrm{LPZ}$ & $9(19 \%)$ & $2(3 \%)$ & 0.008 \\
\hline $\mathrm{MPZ}+\mathrm{CZ}$ & $3(6 \%)$ & 0 & \\
\hline $\mathrm{MPZ}+\mathrm{LPZ}+\mathrm{CZ}$ & $1(2 \%)$ & 0 & \\
\hline
\end{tabular}
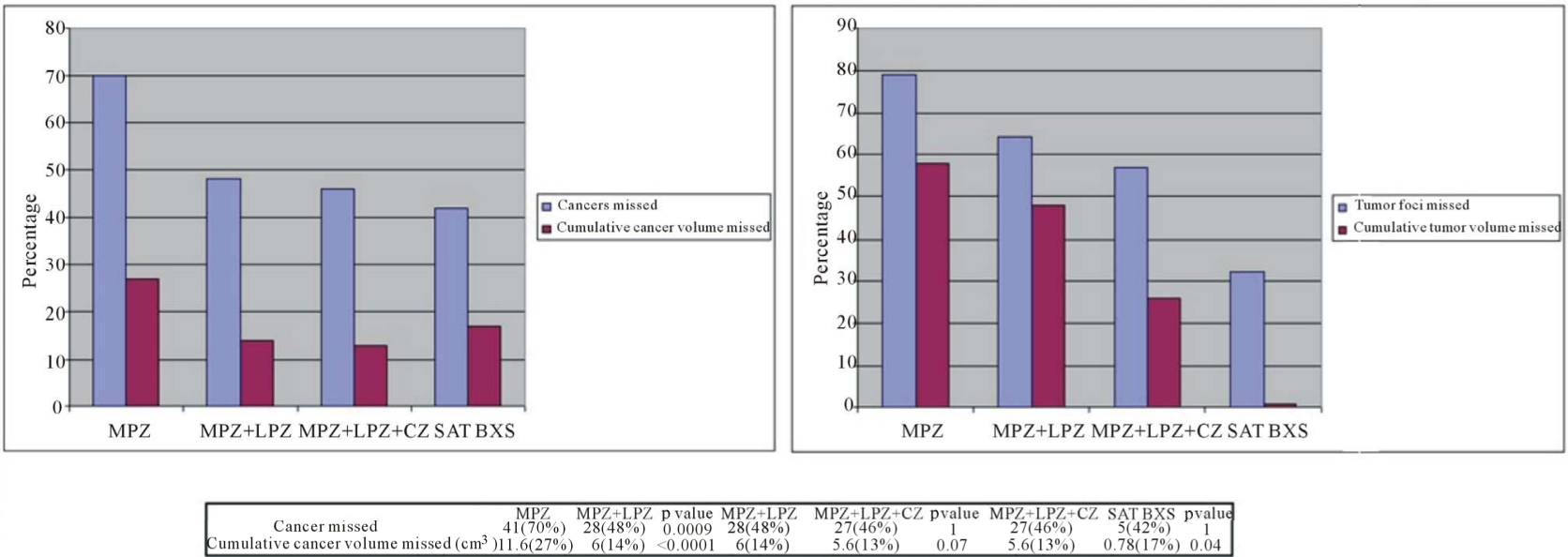

Tumor foci missed

MPZ MPZ+LPZ pvalue MPZ +L

$\begin{array}{lllllll}2 & 0.04\end{array}$

Figure 2. Rates of missed cancers and individual tumor foci, and corresponding volumes, according to biopsy protocol. 


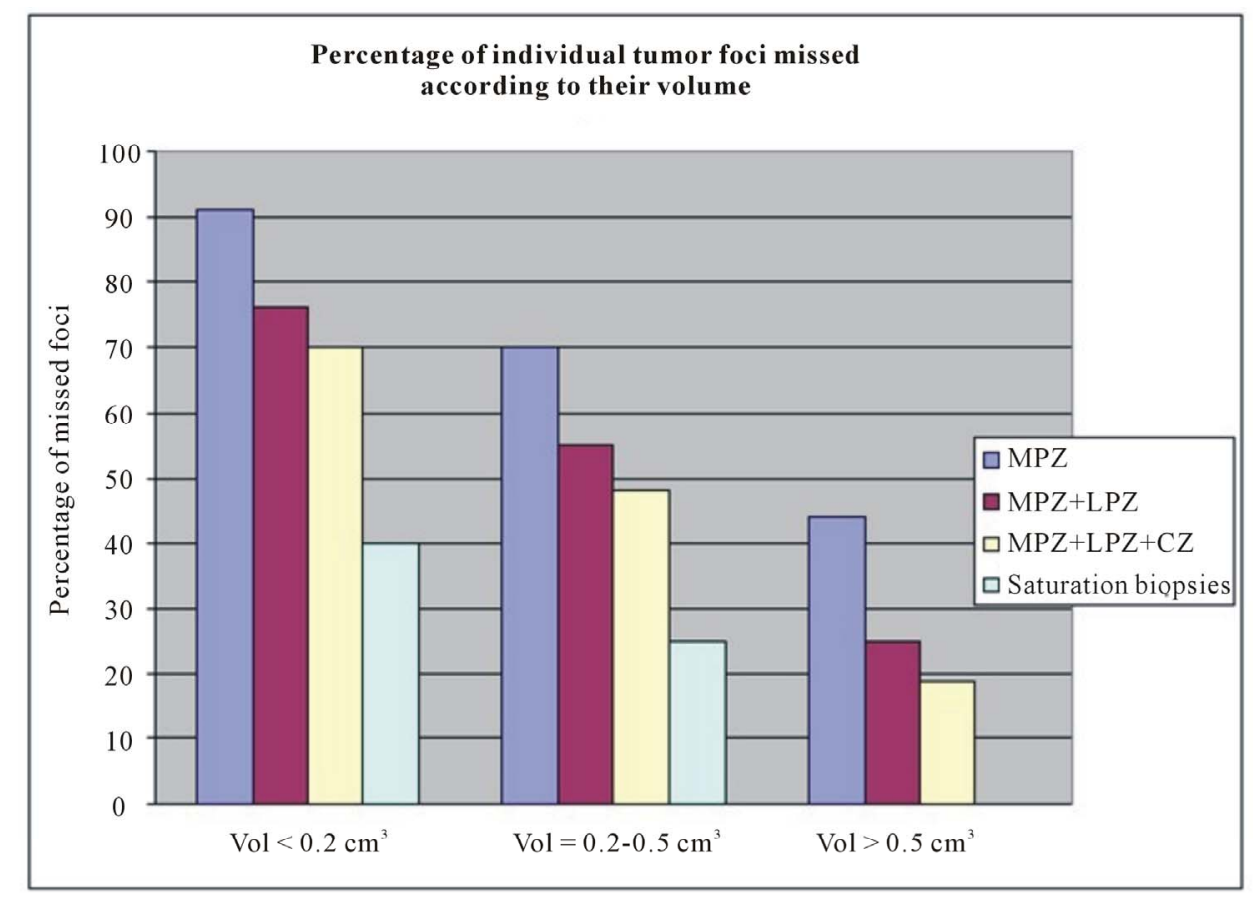

\begin{tabular}{|c|c|c|c|c|c|}
\hline Tumor volume & MPZ & MPZ+LPZ & MPZ+LPZ+CZ & SAT BXS \\
$\leq 0.2 \mathrm{~cm}^{3}$ & $61 / 67(91 \%)$ & $51 / 67(76 \%)$ & $47 / 67(70 \%)$ & $6 / 15(40 \%)$ & P value \\
$0.2-0.5 \mathrm{~cm}^{3}$ & $19 / 27(70 \%)$ & $15 / 27(55 \%)$ & $13 / 27(48 \%)$ & 0.0005 \\
$>0.5 \mathrm{~cm}^{3}$ & $7 / 16(44 \%)$ & $4 / 16(25 \%)$ & $3 / 16(19 \%)$ & 0.04 \\
0
\end{tabular}

Figure 3. Rates of missed individual tumor foci according to their volume.

the clinically significant cancers while missing as much as $48 \%$ of cancers. These missed cancers represented however only $13 \%$ of the overall volume of cancer.

\section{REFERENCES}

[1] K. Eichler, S. Hempel, J. Wilby, et al., "Diagnostic Value of Systematic Biopsy Methods in the Investigation of Prostate Cancer: A Systematic Review," Journal of Urology, Vol. 175, No. 5, 2006, pp. 1605-1612. doi:10.1016/S0022-5347(05)00957-2

[2] V. Scattoni, A. Zlotta, R. Montironi, C. Schulman, P. Rigatti and F. Montorsi, "Extended and Saturation Prostatic Biopsy in the Diagnosis and Characterisation of Prostate Cancer: A Critical Analysis of the Literature," European Urology, Vol. 52, No. 5, 2007, pp. 1309-1322. doi:10.1016/j.eururo.2007.08.006

[3] A. Heidenreich, J. Bellmunt, M. Bolla, et al., "EAU Guidelines on Prostate Cancer. Part 1: Screening, Diagnosis, and Treatment of Clinically Localised Disease," European Urology, Vol. 59, No. 1, 2011, pp. 61-71. doi:10.1016/j.eururo.2010.10.039

[4] T. A. Stamey F. S. Freiha, J. E. McNeal, et al., "Localized Prostate Cancer. Relationship of Tumor Volume to Clinical Significance for Treatment of Prostate Cancer," Cancer, Vol. 71, Supplement S3, 1993, pp. 933-938. doi:10.1002/1097-0142(19930201)71:3+<933::AID-CNC
$\underline{\mathrm{R} 2820711408>3.0 . \mathrm{CO} ; 2-\mathrm{L}}$

[5] J. I. Epstein, P. C. Walsh, M. Carmichael, et al., "Pathologic and Clinical Findings to Predict Tumor Extent of Nonpalpable (T1c) Prostate Cancer," JAMA, Vol. 271, No. 5, 1994, pp. 368-374. doi:10.1001/jama.1994.03510290050036

[6] R. Chou, J. M. Croswell, T. Dana, et al., "Screening for Prostate Cancer: A Review of the Evidence for the US Preventive Services Task Force," Annals of Internal Medicine, Vol. 155, No. 11, 2011, pp. 762-771. doi:10.7326/0003-4819-155-11-201112060-00375

[7] F. H. Schroder, J. Hugosson, M. J. Roobol, et al., "Screening and Prostate-Cancer Mortality in a Randomized European Study," The New England Journal of Medicine, Vol. 360, No. 13, 2009, pp. 1320-1328. doi:10.1056/NEJMoa0810084

[8] F. H. Schröder, J. Hugosson, M. J. Roobol, et al. and ERSPC Investigators, "Prostate-Cancer Mortality at 11 Years of Follow-Up," The New England Journal of Medicine, Vol. 366, No. 11, 2012, pp. 981-990. doi:10.1056/NEJMoa1113135

[9] M. Bul and F. H. Schröder, "Screening for Prostate Cancer-The Controversy Continues, but Can It Be Resolved?" Acta Oncologica, Vol. 50, No. S1, 2011, pp. 4-11. doi:10.3109/0284186X.2010.522197

[10] G. P. Haas, N. B. Delongchamps, R. F. Jones, et al., "Needle Biopsies on Autopsy Prostates: Sensitivity of 
Cancer Detection Based on True Prevalence," Journal of the National Cancer Institute, Vol. 99, No. 19, 2007, pp. 1484-1489. doi:10.1093/jnci/djm153

[11] P. Troncoso, R. J. Babaian, J. Y. Ro, et al., "Prostatic Intraepithelial Neoplasia and Invasive Prostatic Adenocarcinoma in Cystoprostatectomy Specimens," Urology, Vol. 34, Supplement 6, 1989, pp. 52-56.

[12] J. I. Epstein, W. C. Allsbrook Jr., M. B. Amin, et al., "The 2005 International Society of Urological Pathology (ISUP) Concensus Conference on Gleason Grading of Prostatic Carcinoma," The American Journal of Surgical Pathology, Vol. 29, No. 9, 2005, pp. 1228-1242. doi:10.1097/01.pas.0000173646.99337.b1

[13] M. Noguchi, T. A. Stamey, J. E. McNeal, et al., "Assessment of Morphometric Measurements of Prostate Carcinoma Volume," Cancer, Vol. 89, No. 5, 2000, pp. 1056-1064. doi:10.1097/01.pas.0000173646.99337.b1

[14] A. R. Schned, K. J. Wheeler, C. A. Hodorowski, et al., "Tissue-Shrinkage Correction Factor in the Calculation of
Prostate Cancer Volume," The American Journal of Surgical Pathology, Vol. 20, No. 12, 1996, pp. 1501-1506. doi:10.1097/00000478-199612000-00009

[15] A. R. Patel, J. S. Jones, J. Rabets, et al., "Parasagittal Biopsies Add Minimal Information in Repeat Saturation Prostate Biopsy," Urology, Vol. 63, No. 1, 2004, pp. 8789. doi:10.1016/j.urology.2003.08.040

[16] R. K. Nam, R. Saskin, Y. Lee, et al., "Increasing Hospital Admission Rates for Urological Complications after Transrectal Ultrasound Guided Prostate Biopsy," Journal of Urology, Vol. 183, No. 3, 2010, pp. 963-968. doi:10.1016/j.juro.2009.11.043

[17] M. J. Roobol, R. C. N. Van Den Bergh, T. Wolters, et al., "Serum PSA Levels, Number of Prostate Biopsies and Number and Characteristics of Prostate Cancers Detected in Three Consecutive Screening Visits Using a PSA Based Biopsy Indication," European Urology, Vol. 7, No. 3, 2008, p. 199. doi:10.1016/S1569-9056(08)60509-6 